BMJ Open Sport \& Exercise Medicine

\title{
Effect of short moderate intensity exercise bouts on cardiovascular function and maximal oxygen consumption in sedentary older adults
}

\author{
Karani Magutah (D) , ${ }^{1}$ Kihumbu Thairu, ${ }^{2}$ Nilesh Patel ${ }^{2}$
}

\begin{abstract}
To cite: Magutah K, Thairu K, Patel N. Effect of short moderate intensity exercise bouts on cardiovascular function and maximal oxygen consumption in sedentary older adults. BMJ Open Sport \& Exercise Medicine 2020;6:e000672. doi:10.1136/ bmjsem-2019-000672
\end{abstract}

Accepted 6 February 2020

Check for updates

(C) Author(s) (or their employer(s)) 2020. Re-use permitted under CC BY-NC. No commercial re-use. See rights and permissions. Published by BMJ.

${ }^{1}$ Medical Physiology, Moi University School of Medicine, Eldoret, Kenya

${ }^{2}$ Medical Physiology, University of Nairobi, Nairobi, Kenya

Correspondence to Dr Karani Magutah; kmagutah@yahoo.com

\section{ABSTRACT}

Aim To investigate effect of $<10 \mathrm{~min}$ moderate intensity exercise on cardiovascular function and maximal oxygen consumption ( $\dot{V} 0_{2}$ max) among sedentary adults.

Methods We studied 53 sedentary urbanites aged $\geq 50$ years, randomised into: (1) male $\left(\mathrm{M}_{\mathrm{S}}\right)$ and $(2)$ female $\left(\mathrm{F}_{\mathrm{S}}\right)$ undertaking three short-duration exercise (5-10 min) daily, and (3) male $\left(\mathrm{M}_{\mathrm{L}}\right)$ and $(4)$ female $\left(\mathrm{F}_{\mathrm{L}}\right)$ exercising 30-60 min 3-5 days weekly. Resting systolic blood pressure (SBP), diastolic blood pressure (DBP), heart rate and $\dot{V} 0_{2} \max$ were measured at baseline and 8 weekly for 24 weeks. Results At baseline, $50 \% \mathrm{M}_{\mathrm{S}}, 61.5 \% \mathrm{M}_{\mathrm{L}}, 53.8 \% \mathrm{~F}_{\mathrm{S}}$ and $53.8 \% \mathrm{~F}_{\text {f }}$ had $\mathrm{SBP} \geq 120 \mathrm{~mm} \mathrm{Hg}$, and $14.3 \% \mathrm{M}_{\mathrm{S}}, 53.8 \% \mathrm{M}_{\text {, }}$, $23.1 \% \mathrm{~F}_{\mathrm{S}}$ and $38.5 \% \mathrm{~F}$ had $\mathrm{DBP} \geq 80 \mathrm{~mm} \mathrm{Hg}$. At 24 weeks, where SBP remained $\geq 120 \mathrm{~mm} \mathrm{Hg}$, values decreased from $147 \pm 19.2$ to $132.3 \pm 9.6 \mathrm{~mm} \mathrm{Hg}\left(50 \%\right.$ of $\left.M_{S}\right)$, from $144 \pm 12.3$ to $128 \pm 7.0 \mathrm{~mm} \mathrm{Hg}\left(23.1 \%\right.$ of $\left.M_{1}\right)$, from $143.1 \pm 9.6$ to $128.0 \pm 7.0 \mathrm{~mm} \mathrm{Hg}\left(53.8 \%\right.$ of $\left.F_{S}\right)$ and from $152.3 \pm 23.7$ to $129 \pm 3.7 \mathrm{~mm} \mathrm{Hg}\left(30.8 \%\right.$ of $\left.F_{1}\right)$. For DBP $\geq 80 \mathrm{~mm} \mathrm{Hg}, M_{\mathrm{S}}$ and $F_{\mathrm{S}}$ percentages maintained, but values decreased from $101 \pm 15.6$ to $84.5 \pm 0.7 \mathrm{~mm} \mathrm{Hg}\left(\mathrm{M}_{S}\right)$ and $99.0 \pm 3.6$ to $87.7 \pm 4.9 \mathrm{~mm} \mathrm{Hg}\left(F_{S}\right)$. In $M_{L}$ and $F_{L}$, percentage with DBP $\geq 80 \mathrm{~mm} \mathrm{Hg}$ dropped to $15.4 \%$ (86.1 \pm 6.5 to $82.5 \pm 3.5 \mathrm{~mm} \mathrm{Hg})$ and $(91.4 \pm 5.3$ to $83.5 \pm 0.71 \mathrm{~mm} \mathrm{Hg}) . \dot{V}$ $0_{2}$ max increased from $26.1 \pm 4.4$ to $32.0 \pm 6$.2 for $\mathrm{M}_{\mathrm{S}}$, from $25.8 \pm 5.1$ to $28.8 \pm 5.4$ for $M_{1}$ (group differences $p=0.02$ ), from $20.2 \pm 1.8$ to $22.7 \pm 2.0$ for $F_{s}$ and from $21.2 \pm 1.9$ to $24.2 \pm 2.7$ for $F_{1}$ (groups differences $p=0.38$ ).

Conclusion Accumulated moderate intensity exercise bouts of $<10$ min confer similar-to-better cardiovascular and $\dot{V} 0_{2}$ max improvements compared with current recommendations among sedentary adults.

\section{INTRODUCTION}

Recent revision of blood pressure (BP) guidelines has increased the percentage of people in the high BP and hypertension category. ${ }^{12}$ This comes at a time of rising shift in lifestyles where, for example, in Eldoret, Kenya, our study setting, $82 \%$ of the elderly persons do not participate in regular exercise or physical activity (PA) that would result in energy expenditure $\geq 1.5$ metabolic equivalent (MET) minutes, and are therefore sedentary. ${ }^{34}$ Those

\section{What are the new findings?}

- Performing three bouts of moderate intensity exercise each lasting 5-10 min daily yields similarto-better maximal oxygen consumption ( $\begin{aligned} & \dot{V} \\ & 0\end{aligned}$ in sedentary adults compared with current WHO recommendations.

- Accumulating moderate intensity exercise regardless of bout length improves cardiovascular functions of blood pressure and heart rate in both males and females.

in high BP categories also form most of those reported in the current rise in sedentaryrelated health problems in sub-Saharan Africa ${ }^{5-8}$ At a time when health benefits of exercise are being widely promoted, ${ }^{9}$ the reason for decrease in PA, a modifiable risk factor and with it accompanying cardiovascular health problems, is not clear. It is known that high peak maximal oxygen consumption ( $\left.\dot{V} \mathrm{O}_{2} \max \right)$ from increased leisure time $\mathrm{PA}$ is correlated with reduced cardiovascular morbidity and mortality. ${ }^{10}$ Thus, there is a need to change how existing recommendations are promoted, or to develop exercise options which yield similar cardiorespiratory fitness and encourage exercise involvement.

There are many approaches to improve adherence and compliance of PA that have been tested. One approach is the use of shorter exercise sessions, ${ }^{11}$ but its adoption is hindered by lack of sufficient supporting data. Opinions differ on the benefits of short exercise bouts, ${ }^{11-17}$ and a different approach in regime, population or setting that addresses the lack of PA/exercise could be useful. In a previous study involving the same population as in this study, we found that shorter exercise sessions are beneficial in improving cardiometabolic markers and body composition. ${ }^{18} 19$ However, the few studies that have tested the effect of supervised exercise bouts on cardiac performance among the elderly 
have shown conflicting results. ${ }^{20-22}$ Therefore, further studies especially among the lesser studied older sedentary black adults are needed as this group shows $>90 \%$ likelihood of developing hypertension in their lifetimes even when their mid-life BP are normal, ${ }^{23}{ }^{24}$ under the previous BP categorisation that had a higher cut-off point for normal ranges. Thus, in this study, we investigated the effect of moderate intensity jogging bouts lasting $<10 \mathrm{~min}$ on $\mathrm{BP}$, pulse pressure (PP) and heart rate $(\mathrm{HR})$ and $\dot{V}$ $\mathrm{O}_{2}$ max among sedentary black adults who had a weekly MET minutes $<600$ min. $^{9}{ }^{25}$ The study involved $<10 \mathrm{~min}$ bouts in cumulative weekly minutes that matched the current WHO exercise prescription ${ }^{9}$ of $3-5$ weekly sessions each lasting $\geq 30 \mathrm{~min}$.

\section{MATERIALS AND METHODS}

Following a local print advertisement and using the WHO Global Physical Activity Questionnaire (GPAQ) ${ }^{25}$ between September 2016 and May 2017, we recruited 53 (27 males; 26 females) healthy sedentary volunteers aged $\geq 50$ years from Eldoret, Kenya into this randomised controlled field trial. Eligibility for inclusion based on absence of physical injuries, cardiovascular disease (BP screening done at recruitment) or any recent treatment for cardiovascular disease. Hypertensive volunteers were immediately referred for pharmacotherapy. Measurements of $\mathrm{BP}, \mathrm{HR}$ and estimate $\dot{V} \mathrm{O}_{2}$ max were done at the start and at 8 weekly intervals over the course of the study. The shuttle run test (SRT) was used for $\dot{V} \mathrm{O}_{2} \max$ estimation and for continuous cardiovascular assessment during exertion. SRT is a multistage test of cardiovascular fitness and $\dot{V} \mathrm{O}_{2}$ max estimation that incorporates the exercise intensity. This provided the effectiveness of the two different exercise prescriptions. For reproducibility, the same researcher performed all the measurements using the same equipment, protocols, subject posture and time-of-day throughout the 24 weeks of study duration.

Randomisation was done at individual level based on gender and exercise regime, placing participants into four groups: group $1\left(\right.$ male, $\mathrm{M}_{\mathrm{s}}$ ) and group 2 (female, $\mathrm{F}_{\mathrm{s}}$ ) short-duration exercise bouts, and group 3 (male, $\mathrm{M}_{\mathrm{L}}$ ) and group 4 (female, $\mathrm{F}_{\mathrm{L}}$ ) long-duration exercise bouts. The $\mathrm{M}_{\mathrm{S}}$ and $\mathrm{F}_{\mathrm{S}}$ undertook 5-10 min of moderate intensity jogging thrice daily. The $\mathrm{M}_{\mathrm{L}}$ and $\mathrm{F}_{\mathrm{L}}$ undertook the same intensity jogging for 30-60 min 3-5 days per week. All participants kept their own exercise log and wore Polar Wearlink Actitrainer Accelerometers (ActiGraph, Pensacola, Florida, USA) on selected days to assess their adherence to their respective exercise protocol. Exercise logs and accelerometer data were analysed weekly to check if participants had equivalent cumulative exercise durations and MET-minutes.

Data were collected at the start and at 8-week intervals over 24 weeks. For baseline data, participants' biodemographic characteristics, PA and exercise patterns were recorded. Pre-exertion BP was taken as the average of two measurements taken 2 min apart using an electronic sphygmomanometer after $5 \mathrm{~min}$ of rest in a sitting position. PP was determined by getting the difference between the resultant systolic (SBP) and diastolic (DBP) pressures. HR was measured with a polar HR monitor at the start and continuously during the incremental change in speed of the $20 \mathrm{~m}$ SRT up to the point of completion of the test or discontinuation as per the test guidelines. Estimation of $\dot{V} \mathrm{O}_{2}$ max was done by application of the Ramsbottom beep score calculator, ${ }^{26}$ based on the level of the SRT achieved by the participants. Measurements of $\mathrm{HR}$ and $\mathrm{BP}$ were also taken after $5 \mathrm{~min}$ of recovery following completion of the SRT. Thereafter, and for a period of 8 weeks before the next data collection point, participants followed the exercise prescribed for their group by jogging that raised their HR to $50 \%-70 \%$ of their maximal expected HR computed as 220-age in years, one of the criteria for determining moderate intensity exercise. On days when they did not wear accelerometers, this was ascertained by a jog where they could talk but not sing, the accepted alternative where objective monitoring lacks. ${ }^{27}$ Follow-up of participants was done by reminders through phone calls and direct meeting every week. Analysis of completed GPAQs was done every week to check that participants adhered to their exercise prescriptions and that the cumulative exercise times were similar in both groups and for each of the 24 weeks of the protocol. Thus, none of the regimes had a greater improvement than the other and therefore all adhering participants per regime were retained in the study.

STATA V.13 was used for data analysis. Data were presented as mean and SD. Repeated measures analysis of variance was used for analysis of data between the exercise regime groups for each sex over the 24 weeks. A value of $p<0.05$ was considered as statistically significant.

\section{RESULTS}

Mean age for males and females was $55.5 \pm 3.0$ and $53.9 \pm 3.0$ years, respectively. All participants had secondary schoollevel education and $88.4 \%$ of males and $71.4 \%$ of females had university/college education. Ninety-one percentage of participants were currently working in white collar jobs and $9 \%$ had retired from it.

Baseline data showed that $22.2 \%$ of males and $23.1 \%$ of females had pre-SRT BP $\geq 140 / 90 \mathrm{~mm} \mathrm{Hg}$. Those with SBP $>140 \mathrm{~mm} \mathrm{Hg}$ but $\mathrm{DBP}<90 \mathrm{~mm} \mathrm{Hg}$ were $14.8 \%$ and $15.4 \%$ for males and females, respectively. Only one male and one female had DBP $\geq 90 \mathrm{~mm} \mathrm{Hg}$ and SBP $<140 \mathrm{~mm}$ $\mathrm{Hg}$. After SRT at the start, the pre-exercise BP and $5 \mathrm{~min}$ postexercise BP were not different between the participants (SBP: $\mathrm{p}=0.12$ and $\mathrm{p}=0.26$ and DBP: $\mathrm{p}=0.83$ and $\mathrm{p}=0.32$ for males and females, respectively).

Using the lowest cut-offs as per the new BP guidelines for elevated and hypertensive $\mathrm{BP}^{1{ }^{2}} 50 \%$ of $\mathrm{M}_{\mathrm{S}}$ (SBP $147.0 \pm 19.2 \mathrm{~mm} \mathrm{Hg}$ ), $61.5 \%$ of $\mathrm{M}_{\mathrm{L}}$ (SBP $144.0 \pm 12.3 \mathrm{~mm}$ $\mathrm{Hg}), 53.8 \%$ of $\mathrm{F}_{\mathrm{S}}(\mathrm{SBP} 143.1 \pm 9.6 \mathrm{~mm} \mathrm{Hg})$ and $53.8 \%$ of $\mathrm{F}_{\mathrm{L}}$ (SBP $152.3 \pm 23.7 \mathrm{~mm} \mathrm{Hg}$ ) had resting $\mathrm{SBP} \geq 120 \mathrm{~mm} \mathrm{Hg}$ at the start. Using the same guidelines, $14.3 \%$ of $\mathrm{M}_{\mathrm{S}} \mathrm{DBP}$ $(101.0 \pm 15.6 \mathrm{~mm} \mathrm{Hg}), 53.8 \%$ of $\mathrm{M}_{\mathrm{L}}$ (DBP $86.1 \pm 6.5 \mathrm{~mm}$ 
Table 1 Baseline demographic and clinical characteristics of the participants

\begin{tabular}{lcccc}
\hline & $\mathbf{M}_{\mathbf{S}}(\mathbf{n}=\mathbf{1 4})$ & $\mathbf{M}_{\mathbf{L}}(\mathbf{n}=\mathbf{1 3})$ & $\mathbf{F}_{\mathbf{S}}(\mathbf{n}=\mathbf{1 3})$ & $\mathbf{F}_{\mathbf{L}}(\mathbf{n}=\mathbf{1 3})$ \\
\hline Age $($ years $)$ & $55.0 \pm 5.6$ & $55.2 \pm 3.0$ & $53.9 \pm 2.6$ & $53.9 \pm 3.5$ \\
WHtR & $0.52 \pm 0.07$ & $0.56 \pm 0.08$ & $0.61 \pm 0.05$ & $0.57 \pm 0.08$ \\
WHR & $0.93 \pm 0.06$ & $0.96 \pm 0.07$ & $0.82 \pm 0.10$ & $0.84 \pm 0.09$ \\
\hline BMI $\left(\mathrm{kg} / \mathrm{m}^{2}\right)$ & $25.8 \pm 4.0$ & $28.6 \pm 4.8$ & $33.3 \pm 4.8$ & $32.0 \pm 5.4$ \\
\hline FBG $(\mathrm{mmol} / \mathrm{L})$ & $5.9 \pm 0.6$ & $6.4 \pm 2.4$ & $6.1 \pm 0.7$ & $6.2 \pm 0.9$ \\
\hline Body fat percentage & $20.7 \pm 7.3$ & $24.9 \pm 7.9$ & $39.8 \pm 3.6$ & $37.4 \pm 5.1$ \\
\hline Pre-exertion SBP $(\mathrm{mm} \mathrm{Hg})$ & $138.9 \pm 17.4$ & $140.8 \pm 22.1$ & $133.7 \pm 13.2$ & $137.6 \pm 23.8$ \\
\hline Pre-exertion DBP $(\mathrm{mm} \mathrm{Hg})$ & $82.1 \pm 11.3$ & $83.7 \pm 8.7$ & $83.7 \pm 10.6$ & $84.2 \pm 8.1$ \\
\hline Pre-exertion HR $(\mathrm{b} / \mathrm{m})$ & $73.9 \pm 9.5$ & $76.8 \pm 7.7$ & $79.8 \pm 12.0$ & $71.8 \pm 8.4$ \\
\hline Exhaustion SBP $(\mathrm{mm} \mathrm{Hg})$ & $196.3 \pm 17.9$ & $191.6 \pm 20.1$ & $193 \pm 28.0$ & $186.5 \pm 23.2$ \\
\hline Exhaustion DBP $(\mathrm{mm} \mathrm{Hg})$ & $78.7 \pm 7.5$ & $88.7 \pm 8.8$ & $83.3 \pm 11.3$ & $85.5 \pm 9.3$ \\
\hline Exhaustion HR $(\mathrm{b} / \mathrm{m})$ & $125.4 \pm 14.4$ & $127.6 \pm 13.7$ & $125.2 \pm 13.3$ & $123 \pm 17.7$ \\
\hline 5 min postexertion SBP $(\mathrm{mm} \mathrm{Hg})$ & $130.3 \pm 9.8$ & $141 \pm 18.0$ & $127.2 \pm 11.7$ & $128.3 \pm 17.3$ \\
\hline 5 min postexertion DBP $(\mathrm{mm} \mathrm{Hg})$ & $75.4 \pm 9.2$ & $79.5 \pm 9.4$ & $74.1 \pm 10.8$ & $76.1 \pm 10.5$ \\
5 min postexertion HR $(\mathrm{b} / \mathrm{m})$ & $90.8 \pm 8.8$ & $90.8 \pm 11.6$ & $91.5 \pm 10.3$ & $85.1 \pm 8.6$ \\
\hline$\dot{V}$ O max $(\mathrm{mL} / \mathrm{kg} / \mathrm{min})$ & $26.1 \pm 4.4$ & $25.8 \pm 5.1$ & $20.2 \pm 1.8$ & $21.2 \pm 1.9$
\end{tabular}

Data presented as mean \pm SD.

$\mathrm{b} / \mathrm{m}$, beats per minute; DBP, diastolic blood pressure; $F B G$, fasting blood glucose; $F_{L}$, long exercise bouts female; $F_{S}$, short exercise bouts female; HR, heart rate; $M_{L}$, long exercise bouts male; $M_{S}$, short exercise bouts male; SBP, systolic blood pressure; WHR, waist-to-hip ratio; WHtR, waist-to-height ratio.

$\mathrm{Hg}$ ), $23.1 \%$ of $\mathrm{F}_{\mathrm{S}}\left(\mathrm{DBP} 99.0 \pm 3.6 \mathrm{~mm} \mathrm{Hg}\right.$ ) and $38.5 \%$ of $\mathrm{F}_{\mathrm{L}}$ (DBP 91.4+5.3 $\mathrm{mm} \mathrm{Hg}$ ) had DBP $\geq 80 \mathrm{~mm} \mathrm{Hg}$. Similarly, all groups had $\dot{V} \mathrm{O}_{2}$ max lower than expected for their ages. Based on existing categorisations for gender and age, $\mathrm{M}_{\mathrm{S}}$, $\mathrm{M}_{\mathrm{L}}$ and $\mathrm{F}_{\mathrm{S}}$ groups had 'very poor' levels of $\dot{\mathrm{V}} \mathrm{O}_{2}$ max with the $\mathrm{F}_{\mathrm{L}}$ group in the 'poor' category. ${ }^{28}$ Accelerometry data showed weekly cumulative exercise minutes to be similar for $\mathrm{M}_{\mathrm{S}}$ and $\mathrm{M}_{\mathrm{L}}(161.8 \pm 7.2$ vs $162.6 \pm 6.1)$, and also for $\mathrm{F}_{\mathrm{S}}$ and $\mathrm{F}_{\mathrm{L}}$ (158.3 \pm 3.6 vs 156.1 \pm 2.7 , respectively). Adherence over the 24 weeks regime was $100 \%$ for $\mathrm{M}_{\mathrm{S}}(\mathrm{n}=14)$ and $\mathrm{F}_{\mathrm{S}}(\mathrm{n}=13)$, and $61.5 \%$ and $76.9 \%$ for $\mathrm{M}_{\mathrm{L}}(\mathrm{n}=14)$ and $\mathrm{F}_{\mathrm{L}}$ $(\mathrm{n}=13)$, respectively. These baseline demographic and clinical parameters are shown in table 1.

At the 24th week, although $50 \%$ of $\mathrm{M}_{\mathrm{s}}$ group had SBP $\geq 120 \mathrm{~mm} \mathrm{Hg}$, their mean SBP decreased from $147 \pm 19.2 \mathrm{~mm} \mathrm{Hg}$ to $132.3 \pm 9.6 \mathrm{~mm} \mathrm{Hg}$. In the $\mathrm{M}_{\mathrm{L}}$ group, the percentage with $\mathrm{SBP} \geq 120 \mathrm{~mm} \mathrm{Hg}$ dropped from $61.5 \%$ to $23.1 \%(144 \pm 12.3 \mathrm{~mm} \mathrm{Hg}$ to $128 \pm 7.0 \mathrm{~mm} \mathrm{Hg})$. Although the percentage with $\mathrm{SBP} \geq 120 \mathrm{~mm} \mathrm{Hg}$ in $\mathrm{F}_{\mathrm{S}}$ group did not change, the mean values decreased from $143.1 \pm 9.6 \mathrm{~mm} \mathrm{Hg}$ to $128.0 \pm 7.0 \mathrm{~mm} \mathrm{Hg}$. In the $\mathrm{F}_{\mathrm{L}}$ group, the percentage with $\mathrm{SBP} \geq 120 \mathrm{~mm} \mathrm{Hg}$ dropped from $53.8 \%$ to $30.8 \%(152.3 \pm 23.7 \mathrm{~mm} \mathrm{Hg}$ to $129 \pm 3.7 \mathrm{~mm} \mathrm{Hg})$. For those with DBP $\geq 80 \mathrm{~mm} \mathrm{Hg}$, there was no percentage change in either the $\mathrm{M}_{\mathrm{S}}$ or $\mathrm{F}_{\mathrm{S}}$ groups, but their mean values dropped from $101 \pm 15.6 \mathrm{~mm} \mathrm{Hg}$ to $84.5 \pm 0.7 \mathrm{~mm}$ $\mathrm{Hg}$ and from $99.0 \pm 3.6 \mathrm{~mm} \mathrm{Hg}$ to $87.7 \pm 4.9 \mathrm{~mm} \mathrm{Hg}$, respectively. For the $\mathrm{M}_{\mathrm{L}}$ group, the percentage with DBP $\geq 80 \mathrm{~mm} \mathrm{Hg}$ dropped from $53.8 \%$ to $15.4 \%$ ( $86.1 \pm 6.5 \mathrm{~mm}$ $\mathrm{Hg}$ to $82.5 \pm 3.5 \mathrm{~mm} \mathrm{Hg}$ ), and from $38.5 \%$ to $15.4 \%$ in the
$\mathrm{F}_{\mathrm{L}}$ group $(91.4 \pm 5.3 \mathrm{~mm} \mathrm{Hg}$ to $83.5 \pm 0.71 \mathrm{~mm} \mathrm{Hg})$. Pooling data from all participants, we similarly observed a mixed decrease in $\mathrm{BP}$ and $\mathrm{HR}$ in the two exercise groups. Resting $\mathrm{SBP}$ in $\mathrm{M}_{\mathrm{L}}$, resting DBP in $\mathrm{M}_{\mathrm{S}}$ as well as DBP at $5 \mathrm{~min}$ in $\mathrm{M}_{\mathrm{S}}$ all reduced significantly $(\mathrm{p}<0.05)$. These significant declines however showed no difference in the manner of the change between regimes (all $\mathrm{p} \geq 0.05$ ) (table 2). Comparison of changes in different variables between study groups showed no significant gender differences. Associated PP changes over the study period are shown in table 3. Except for PP decay at exhaustion among males, there was no differences between the exercise groups. This was despite $\mathrm{M}_{\mathrm{L}}$ resting $\mathrm{PP}$ and $\mathrm{F}_{\mathrm{S}}$ exhaustion PP showing significant improvement over the 24 weeks compared with respective opposite regimes. For the two regimes, the pre-SRT and 5 min post-SRT BP differences were the same (SBP: $p=0.42$ and $p=0.24$ and DBP: $\mathrm{p}=0.83$ and $\mathrm{p}=0.32$, for males and females, respectively).

The $\dot{V} \mathrm{O}_{2}$ max across all groups rose from baseline to the 24th week (figure 1), but there was no significant difference between the groups of the same sex at each of the four data collection points $(p \geq 0.05)$ for the different exercise regimes. The mean change in $\dot{V} \mathrm{O}_{2}$ max at the start and 24th week were however different among males $(\mathrm{p}=0.02$ ) but not females (figure 2$)$.

\section{DISCUSSION}

\section{Baseline results}

At the start of the study, the participants' mean preexertion SBP and DBP were in the stage 1 hypertension 
Table 2 Absolute blood pressure, heart rate and $\mathrm{V} \cdot \dot{\mathrm{V}} \mathrm{O}_{2}$ max changes

\begin{tabular}{|c|c|c|c|c|c|c|c|c|}
\hline Variable & Group & Week 0 & Week 8 & Week 16 & Week 24 & $\begin{array}{l}\text { ( } \Delta \text { from } \\
\text { baseline) }\end{array}$ & $\begin{array}{l}\Delta \text { (week } \\
\text { 24-week 0) }\end{array}$ & $\begin{array}{l}P \\
\text { value }\end{array}$ \\
\hline \multicolumn{9}{|l|}{ Male } \\
\hline \multirow[t]{2}{*}{ Resting SBP } & $M_{s}$ & $138.9 \pm 17.4$ & $132.9 \pm 15.2$ & $130.6 \pm 16.9$ & $122.4 \pm 12.5$ & 0.25 & $-16.4 \pm 10.7$ & \\
\hline & $M_{L}$ & $138.6 \pm 26.4$ & $131.9 \pm 25.1$ & $126.6 \pm 15.1$ & $116.5 \pm 10.7$ & 0.03 & $-22.1 \pm 27.6$ & 0.49 \\
\hline Exhaustion SBP & $M_{s}$ & $196.3 \pm 17.9$ & $194.8 \pm 21.8$ & $189.6 \pm 21.3$ & $177.5 \pm 14.5$ & 0.46 & $-18.8 \pm 11.6$ & \\
\hline \multirow[t]{2}{*}{$\mathrm{SBP}$ at $5 \mathrm{~min}$} & $M_{L}$ & $185.9 \pm 20.6$ & $183.6 \pm 19.6$ & $176.1 \pm 13.2$ & $175.6 \pm 15.3$ & 0.45 & $-10.3 \pm 10.1$ & 0.1 \\
\hline & $M_{s}$ & $130.3 \pm 9.8$ & $132.1 \pm 14.0$ & $129.1 \pm 15.0$ & $120.7 \pm 8.1$ & 0.52 & $-9.6 \pm 10.1$ & \\
\hline \multirow[t]{2}{*}{ Resting DBP } & $M_{L}$ & $137.6 \pm 16.8$ & $131.5 \pm 9.1$ & $125.4 \pm 12.0$ & $117.9 \pm 7.5$ & 0.05 & $-19.9 \pm 16.5$ & 0.08 \\
\hline & $M_{s}$ & $82.1 \pm 11.3$ & $78.5 \pm 10.6$ & $74.0 \pm 8.8$ & $71.8 \pm 5.8$ & 0.02 & $-10.4 \pm 7.8$ & \\
\hline Exhaustion DBP & $M_{L}$ & $83.0 \pm 10.0$ & $80.8 \pm 12.7$ & $76.4 \pm 8.5$ & $74.0 \pm 6.7$ & 0.31 & $-9.0 \pm 7.4$ & 0.69 \\
\hline \multirow[t]{2}{*}{$\mathrm{DBP}$ at $5 \mathrm{~min}$} & $M_{S}$ & $78.7 \pm 7.5$ & $80.1 \pm 7.1$ & $79.0 \pm 8.6$ & $75.4 \pm 5.4$ & 0.25 & $-3.3 \pm 6.0$ & \\
\hline & $M_{L}$ & $86.8 \pm 9.7$ & $84.4 \pm 5.6$ & $83.5 \pm 5.3$ & $78.5 \pm 4.5$ & 0.06 & $-8.3 \pm 8.5$ & 0.12 \\
\hline \multirow[t]{2}{*}{ Resting HR } & $M_{s}$ & $75.4 \pm 9.2$ & $72.5 \pm 6.2$ & $71.3 \pm 7.2$ & $67.8 \pm 4.2$ & 0.01 & $-7.6 \pm 7.6$ & \\
\hline & $M_{L}$ & $77.8 \pm 4.9$ & $78.1 \pm 3.6$ & $74.1 \pm 6.4$ & $73.0 \pm 3.8$ & 0.52 & $-4.8 \pm 7.2$ & 0.39 \\
\hline \multirow[t]{2}{*}{ Exhaustion HR } & $M_{s}$ & $73.9 \pm 9.5$ & $70.0 \pm 9.4$ & $66.1 \pm 9.9$ & $63.0 \pm 7.2$ & 0.34 & $-10.9 \pm 8.6$ & \\
\hline & $M_{L}$ & $74.9 \pm 8.3$ & $71.0 \pm 6.6$ & $72.0 \pm 6.9$ & $64.8 \pm 5.7$ & 0.35 & $-10.1 \pm 8.1$ & 0.85 \\
\hline \multirow[t]{2}{*}{$\mathrm{HR}$ at $5 \mathrm{~min}$} & $M_{s}$ & $125.4 \pm 14.4$ & $120.5 \pm 17.0$ & $113.6 \pm 18.3$ & $118.4 \pm 15.4$ & 0.81 & $-6.9 \pm 15.5$ & \\
\hline & $M_{L}$ & $129.1 \pm 14.7$ & $119.8 \pm 20.1$ & $125.9 \pm 14.9$ & $130.3 \pm 16.7$ & 0.74 & $1.1 \pm 14.0$ & 0.24 \\
\hline \multirow[t]{2}{*}{$\dot{V} \mathrm{O}_{2} \max$} & $M_{S}$ & $90.8 \pm 8.8$ & $85.9 \pm 11.0$ & $82.4 \pm 7.6$ & $74.9 \pm 5.6$ & 0.12 & $-15.9 \pm 7.9$ & \\
\hline & $M_{L}$ & $91.4 \pm 13.0$ & $84.0 \pm 10.1$ & $81.5 \pm 12.5$ & $76.9 \pm 7.6$ & 0.18 & $-14.5 \pm 10.3$ & 0.73 \\
\hline Female & $M_{s}$ & $26.1 \pm 4.4$ & $28.4 \pm 5.7$ & $29.7 \pm 6.0$ & $32.0 \pm 6.2$ & 0.23 & $5.88 \pm 2.8$ & \\
\hline Resting SBP & $M_{L}$ & $25.8 \pm 5.1$ & $26.1 \pm 5.2$ & $27.1 \pm 5.2$ & $28.8 \pm 5.4$ & 0.89 & $2.96 \pm 2.0$ & 0.02 \\
\hline Exhaustion SBP & $\mathrm{F}_{\mathrm{s}}$ & $133.7 \pm 13.2$ & $129.9 \pm 15.4$ & $125.5 \pm 14.8$ & $118.5 \pm 12.8$ & 0.91 & $-15.2 \pm 4.1$ & \\
\hline \multirow[t]{2}{*}{$\mathrm{SBP}$ at $5 \mathrm{~min}$} & $\mathrm{~F}_{\mathrm{L}}$ & $130.5 \pm 16.1$ & $126.5 \pm 14.5$ & $122.6 \pm 15.2$ & $116.4 \pm 11.4$ & 0.31 & $-14.1 \pm 11.8$ & 0.75 \\
\hline & $\mathrm{F}_{\mathrm{s}}$ & $193.0 \pm 28.0$ & $182.7 \pm 24.0$ & $178.2 \pm 25.1$ & $174.7 \pm 25.4$ & 0.74 & $-18.3 \pm 13.8$ & \\
\hline \multirow[t]{2}{*}{ Resting DBP } & $\mathrm{F}_{\mathrm{L}}$ & $182.0 \pm 24.5$ & $178.4 \pm 27.8$ & $170.8 \pm 15.7$ & $167.2 \pm 16.6$ & 0.26 & $-14.8 \pm 19.5$ & 0.62 \\
\hline & $\mathrm{F}_{\mathrm{s}}$ & $127.2 \pm 11.7$ & $124.2 \pm 16.5$ & $124.6 \pm 12.2$ & $119.5 \pm 14.1$ & 0.53 & $-7.6 \pm 8.4$ & \\
\hline Exhaustion DBP & $\mathrm{F}_{\mathrm{L}}$ & $122.5 \pm 13.2$ & $121.8 \pm 14.8$ & $118.5 \pm 11.0$ & $114.3 \pm 11.0$ & 0.6 & $-8.2 \pm 12.2$ & 0.89 \\
\hline \multirow[t]{2}{*}{$\mathrm{DBP}$ at $5 \mathrm{~min}$} & $\mathrm{~F}_{\mathrm{s}}$ & $83.7 \pm 10.6$ & $80.1 \pm 11.2$ & $77.2 \pm 10.3$ & $73.9 \pm 9.0$ & 0.56 & $-9.8 \pm 5.5$ & \\
\hline & $\mathrm{F}_{\mathrm{L}}$ & $81.8 \pm 7.6$ & $75.9 \pm 8.6$ & $75.3 \pm 7.6$ & $70.8 \pm 8.0$ & 0.89 & $-11.0 \pm 4.6$ & 0.57 \\
\hline \multirow[t]{2}{*}{ Resting HR } & $\mathrm{F}_{\mathrm{s}}$ & $83.3 \pm 11.3$ & $84.2 \pm 10.2$ & $83.8 \pm 11.1$ & $80.5 \pm 9.5$ & 0.57 & $-2.8 \pm 8.9$ & \\
\hline & $\mathrm{F}_{\mathrm{L}}$ & $84.4 \pm 9.5$ & $81.7 \pm 10.6$ & $79.7 \pm 7.1$ & $75 \pm 6.3$ & 0.24 & $-9.4 \pm 8.4$ & 0.08 \\
\hline \multirow[t]{2}{*}{ Exhaustion HR } & $\mathrm{F}_{\mathrm{s}}$ & $74.1 \pm 10.8$ & $75.5 \pm 10.6$ & $73.6 \pm 8.3$ & $70.5 \pm 8.2$ & 0.36 & $-3.6 \pm 6.1$ & \\
\hline & $\mathrm{F}_{\mathrm{L}}$ & $74.6 \pm 10.9$ & $74.6 \pm 19.9$ & $69.4 \pm 8.7$ & $68.1 \pm 6.5$ & 0.13 & $-6.5 \pm 7.0$ & 0.3 \\
\hline \multirow[t]{2}{*}{$\mathrm{HR}$ at $5 \mathrm{~min}$} & $\mathrm{~F}_{\mathrm{s}}$ & $79.8 \pm 12.0$ & $73.9 \pm 12.4$ & $71.7 \pm 7.9$ & $66.4 \pm 6.5$ & 0.05 & $-13.4 \pm 11.3$ & \\
\hline & $\mathrm{F}_{\mathrm{L}}$ & $68.2 \pm 5.6$ & $68.2 \pm 11.4$ & $64.0 \pm 7.2$ & $62.8 \pm 7.7$ & 0.36 & $-5.4 \pm 4.5$ & 0.05 \\
\hline \multirow[t]{6}{*}{$\dot{\mathrm{V}} \mathrm{O}_{2} \max$} & $\mathrm{F}_{\mathrm{s}}$ & $125.2 \pm 13.3$ & $123.1 \pm 15.8$ & $121.3 \pm 19.6$ & $119.8 \pm 14.3$ & 0.82 & $-5.4 \pm 13.8$ & \\
\hline & $\mathrm{F}_{\mathrm{L}}$ & $122.7 \pm 19.1$ & $113.3 \pm 17.5$ & $112.6 \pm 21.4$ & $116.9 \pm 21.5$ & 0.73 & $-5.8 \pm 21.3$ & 0.96 \\
\hline & $\mathrm{F}_{\mathrm{s}}$ & $91.5 \pm 10.3$ & $86.3 \pm 6.2$ & $81.7 \pm 7.1$ & $75.7 \pm 6.9$ & 0.18 & $-15.8 \pm 9.3$ & \\
\hline & $\mathrm{F}_{\mathrm{L}}$ & $84.2 \pm 9.6$ & $81.6 \pm 10.1$ & $73.6 \pm 6.5$ & $71.3 \pm 5.7$ & 0.13 & $-12.9 \pm 8.9$ & 0.46 \\
\hline & $\mathrm{F}_{\mathrm{s}}$ & $20.2 \pm 1.8$ & $20.7 \pm 1.9$ & $21.2 \pm 2.1$ & $22.7 \pm 2.0$ & 0.71 & $2.51 \pm 1.0$ & \\
\hline & $\mathrm{F}_{\mathrm{L}}$ & $21.2 \pm 1.9$ & $22.1 \pm 2.9$ & $23.0 \pm 2.8$ & $24.2 \pm 2.7$ & 0.32 & $2.98 \pm 1.5$ & 0.38 \\
\hline
\end{tabular}

DBP, diastolic blood pressure; $F_{L}$, long exercise bouts female; $F_{S}$, short exercise bouts female; HR, heart rate; $M_{L}$, long exercise bouts male; $M_{S}$, short exercise bouts male; SBP, systolic blood pressure. 
Table 3 Pulse pressure changes over 24 weeks of the study period

\begin{tabular}{|c|c|c|c|c|c|c|c|c|}
\hline Variable & Group & Baseline & Week 8 & Week 16 & Week 24 & $\begin{array}{l}\text { P value } \\
\text { ( } \Delta \text { from } \\
\text { baseline) }\end{array}$ & $\begin{array}{l}\text { Mean } \Delta \\
\text { (week 24- } \\
\text { week 0) }\end{array}$ & $P$ value \\
\hline \multirow[t]{2}{*}{ Resting PP } & $\mathrm{M}_{\mathrm{S}}$ & $56.7 \pm 11.4$ & $54.4 \pm 7.5$ & $56.6 \pm 11.5$ & $50.6 \pm 9.1$ & 0.43 & $-6.1 \pm 10.8$ & \\
\hline & $M_{L}$ & $55.6 \pm 17.7$ & $51.1 \pm 17.2$ & $50.3 \pm 8.7$ & $42.5 \pm 7.1$ & 0.03 & $-13.1 \pm 22.2$ & 0.32 \\
\hline \multirow[t]{2}{*}{5 min rest $\mathrm{PP}$} & $\mathrm{M}_{\mathrm{S}}$ & $54.9 \pm 9.7$ & $59.6 \pm 12.0$ & $57.8 \pm 11.2$ & $53.0 \pm 7.3$ & 0.32 & $-1.9 \pm 10.2$ & \\
\hline & $M_{L}$ & $60.0 \pm 17.1$ & $53.4 \pm 6.6$ & $51.3 \pm 7.0$ & $44.9 \pm 8.1$ & 0.07 & $-15.1 \pm 19.0$ & 0.05 \\
\hline \multicolumn{9}{|l|}{ Female } \\
\hline Resting PP & $\mathrm{F}_{\mathrm{S}}$ & $50.0 \pm 7.1$ & $49.8 \pm 8.4$ & $48.3 \pm 6.4$ & $44.5 \pm 7.7$ & 0.77 & $-5.5 \pm 4.8$ & \\
\hline \multirow[t]{2}{*}{5 min rest $\mathrm{PP}$} & $\mathrm{F}_{\mathrm{S}}$ & $53.1 \pm 6.7$ & $48.7 \pm 9.7$ & $51.0 \pm 7.1$ & $49.1 \pm 8.1$ & 0.51 & $-4.0 \pm 7.4$ & \\
\hline & $\mathrm{F}_{\mathrm{L}}$ & $47.9 \pm 12.2$ & $47.2 \pm 10.8$ & $49.1 \pm 8.1$ & $46.2 \pm 6.7$ & 0.09 & $-1.7 \pm 11.4$ & 0.56 \\
\hline
\end{tabular}

Values are means $\pm S D$. $P<0.05$ : significant difference in mean change between the two regimes.

$F_{L}$, long exercise bouts female; $F_{S}$, short exercise bouts female; $M_{L}$, long exercise bouts male; $M_{S}$, short exercise bouts male; PP, pulse pressure.

category based on the current BP guidelines, ${ }^{12}$ that is, between 130-139 SBP and 80-89 DBP mm Hg. Thus, a majority of participants had hypertensive tendency at recruitment. Their SBP rise and DBP decay at exhaustion from SRT, and return to pre-exertion values within 5 min of rest was within previously reported data. ${ }^{29}$ That the $5 \mathrm{~min}$ post-SRT BP was similar to the BP before the run test demonstrated good normalisation. However, as nearly a quarter had pre-exertion BP $\geq 140 / 90$, and, based on the WHO and the criterion by Alberti et al for including SBP $\geq 130 \mathrm{~mm} \mathrm{Hg}$ or DBP $\geq 85 \mathrm{~mm} \mathrm{Hg}$ in metabolic syndrome diagnosis, ${ }^{30} 31$ and, further, given the new published guidelines, ${ }^{12}$ a significant proportion of our participants appeared at risk to developing metabolic syndrome at the start. Similarly, based on the published reference $\dot{V} \mathrm{O}_{2} \max$ values, ${ }^{28}$ the $\dot{V} \mathrm{O}_{2} \max$ means for males and females at the start were below that for their ages, demonstrating a reduced cardiorespiratory health probably related or due to their sedentary lifestyle. With advancing age and adoption of sedentary lifestyles, the

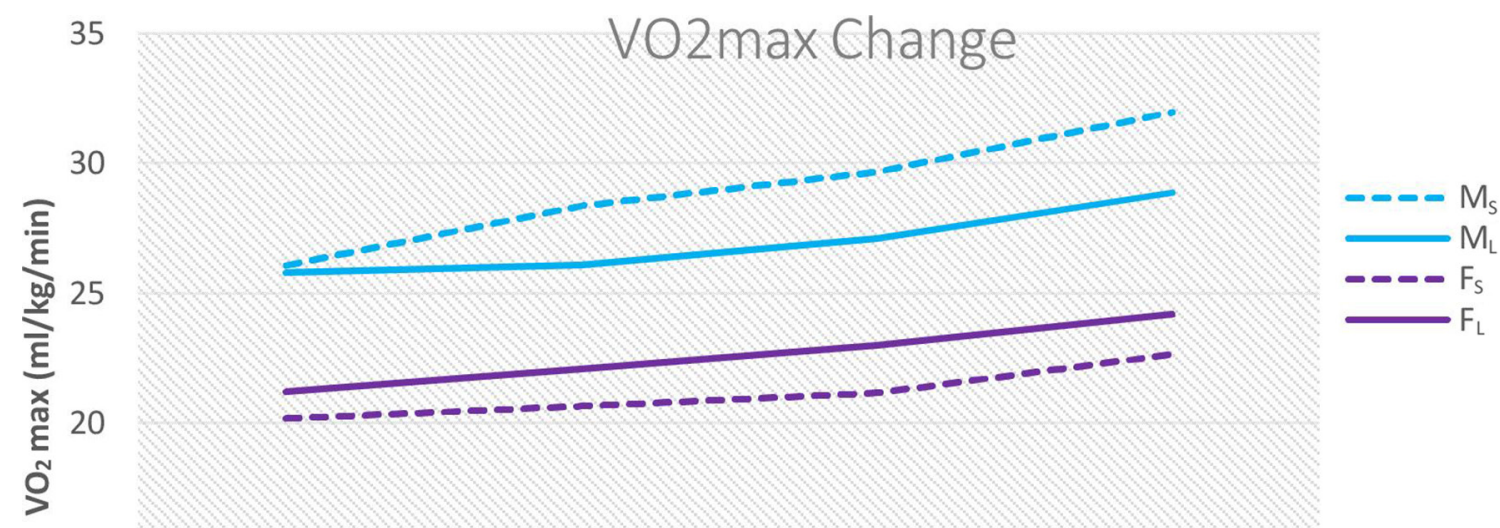

15

10

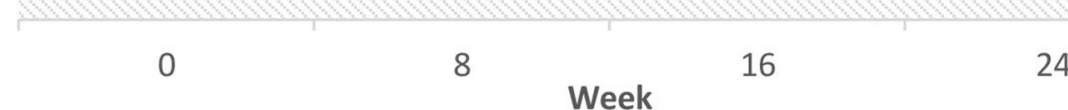

Week

24

Figure $1 \dot{\mathrm{V}} \mathrm{O}_{2}$ max from week 1 to week $24 . \mathrm{M}_{\mathrm{S}}$, short exercise bouts male; $\mathrm{M}_{\mathrm{L}}$, long exercise bouts male; $\mathrm{F}_{\mathrm{S}}$, short exercise bouts female; $F_{L}$, long exercise bouts female. 
Mean VO2max Change: Baseline and Week 24

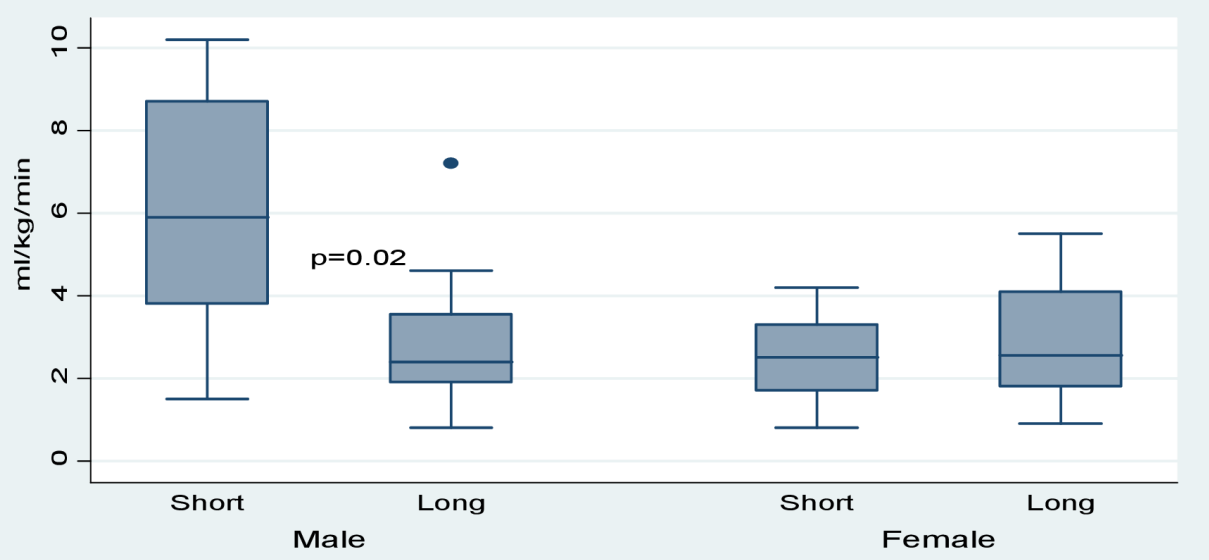

Figure 2 Mean change in $\dot{V} \mathrm{O}_{2}$ max between the 1st and 24th week. Short, short exercise bouts; long, long exercise bouts. $\mathrm{P}=0.02$ denotes significant difference between short and long exercise bouts among males.

low $\dot{V} \mathrm{O}_{2}$ max and elevated BP pose higher risk for cardiovascular disease. $^{12223233}$

\section{Cardiovascular effects at end point}

All the participants in the different exercise groups showed mixed decrease in BP and HR over the 24-week exercise period. The BP and HR levels before the SRT, at exhaustion from the SRT and at $5 \mathrm{~min}$ after SRT appeared to decline over the study period. Among $M_{L}$, resting SBP, resting $\mathrm{DBP}$ and $\mathrm{DBP}$ at $5 \mathrm{~min}$ all showed significant drop at week 24 when compared with respective baseline values. What was novel is that the mean change difference between week 24 and baseline was similar for the two regimes. While exercise has been shown to improve cardiovascular performance, thus reducing cardiovascular mortality and morbidity among the elderly through reduction of fatty deposits in small blood vessel walls that cause blockage and/or reduced elasticity thus raising $\mathrm{BP},{ }^{34-38}$ our findings show that short exercise bouts were as effective in reducing BP and HR as the currently recommended longer exercise bouts, ${ }^{9}$ although the follow-up period probably needed lengthening to demonstrate significant changes in more variables. Until recently, when Landram et $a l^{39}$ demonstrated that intermittent bouts of $<10 \mathrm{~min}$ had comparable BP outcomes as the recommended exercise regime, ${ }^{39}$ not much was known of what effect $<30$ min exercise bouts would have on cardiovascular parameters. In that study, unlike this study, the age range was broader, and the rest interval between the short exercise bouts was $10 \mathrm{~min}$. Another study ${ }^{40}$ used longer exercise bouts of $15 \mathrm{~min}$, but we found a similar reduction in $\mathrm{BP}$ and HR using shorter bouts.

After each SRT, no difference was found in HR recovery to the pre-SRT levels in the different exercise groups over the 24-week study period. Furthermore, in all exercise groups, HR both before SRT and after the $5 \mathrm{~min}$ post-SRT rest period was lower at the end of 24 th week compared with the start. Additionally, the HR recovery following SRT at 24th week was similar for the two regimes. The similarity in the HR recovery after SRT at the end of the study underscores the similarity of effects of intermittent exercise regime tested in this study. This shows that the shorter bouts could be as effective in producing the benefits associated with faster and near complete HR recovery for better cardiovascular health, ${ }^{41}{ }^{42}$ as is seen with the traditional (longer bout) regimes.

$\mathrm{PP}$ is an accepted indicator of cardiovascular mortality and morbidity with lower values being protective. ${ }^{43-45}$ In our study, resting PP dropped over 24 weeks in both males and females for both exercise regimes, but, except in exhaustion PP where mean change was higher in $M_{S}$ compared with $M_{L}$, there was no difference in the mean change between regimes. Start to end point data showed significant drops only in resting PP among $M_{L}$ and exhaustion PP among $\mathrm{F}_{\mathrm{L}}$, but the study had primarily sought to test for differences in the mean change over the study period between the regimes, which was found to be largely similar. This mix of results suggest that none of the regimes is superior. Recent studies have shown workouts lasting $>45 \mathrm{~min}$ for a period of 8 weeks duration reduce $\mathrm{PP},{ }^{46}$ and our study shows that exercise bouts lasting $<10 \mathrm{~min}$, performed regularly, are also effective in reducing the PP. However, the proposed threshold for improved PP, that is, $<76 \mathrm{~mm} \mathrm{Hg},{ }^{45}$ was not achieved in this study, and probably a longer period of exercise may further improve PP.

The similarity of change in SBP, DBP, HR and PP in all the groups shows that in this population, short exercise bouts are as effective as the longer ones in improving these parameters. Based on this finding, the short exercise bouts could be used for individuals of similar demographics.

\section{$\dot{V} 0_{2} \max$ effects at end point}

In line with the reported increase in $\dot{V} \mathrm{O}_{2} \max$ with moderate intensity exercise, ${ }^{47-49} \dot{V} \mathrm{O}_{2} \max$ appeared to increase in both males and females over the 24 weeks study period, and probably a longer follow-up may probably have yielded more significant changes. There however was a significant difference in the manner of $\dot{V}$ 
$\mathrm{O}_{2}$ max change for males but not for the females. Males in the accumulated shorter exercise bouts showed higher $\dot{V} \mathrm{O}_{2}$ max compared with those on the longer exercise bouts. It has been demonstrated that short exercise bouts may be better in improving $\dot{V} \mathrm{O}_{2}$ max. ${ }^{40}$

As the rate of increase of $\dot{V} \mathrm{O}_{2}$ max over the 24 weeks showed either no difference between the short or long exercise bouts in the females, or, that indeed short bouts were better for males suggests that the effect of accumulated short bouts exercise on $\dot{V} \mathrm{O}_{0} \max$ is at least similar to, if not better than the currently recommended exercise bouts lasting $\geq 30 \mathrm{~min}$. Similar results have been reported in a short 4-week study where individuals with cumulatively similar weekly exercise time with exercise regimes of 10 and $30 \mathrm{~min}$ per session, showed no difference in $\dot{V} \mathrm{O}_{2}$ max change. ${ }^{39}$ Our findings add to the growing evidence that intermittent exercise programme performed so that the cumulative exercise time matches the current recommended exercise regime is at least as effective as the existing recommendation of 150 weekly minutes of moderate intensity exercise done in $3-5$ weekly sessions of $30-60$ min each, ${ }^{9}$ in increasing $\dot{V}$ $\mathrm{O}_{2}$ max among the elderly.

However, none of the exercise regimes used in this study helped participants achieve $\dot{V} \mathrm{O}_{2}$ max levels for their age. While there was improvement for all groups, the $\dot{V}$ $\mathrm{O}_{2}$ max values remained below the proposed levels that correspond to better Cardiorespiratory fitness outcomes. In fact, across all our groups, the improvement over the 24-week period was marginal, rising from the very poor and poor categories to at the most fair category as compared with the Physical Fitness Certification Manual ${ }^{28}$ information. A longer exercise periods may yield better improvements for this age bracket whose bodies may take longer to adjust and who have higher loss in aerobic power, ${ }^{50}$ compared with the younger populations.

\section{Limitations}

Participants self-selected themselves for this study in response to a local print advert. This could have affected the representativeness of results in that the recruitment criteria were biased towards those who saw the print advert only. Additionally, as it was impossible to include blinding in the study protocol, the investigators and the participants all knew who was on which exercise regime. This may have affected adherence from peer influences. Furthermore, dietary habits, use of stimulants like caffeine and participants' renal status, all known confounders in cardiovascular and $\dot{V} \mathrm{O}_{2} \max$ measurements were not considered during the follow-up and the data analysis. That we used indirect methods for $\dot{V} \mathrm{O}_{2}$ max estimation may also have affected our results. These confounders may affect the generalisability of our results.

\section{Strengths}

Our study showed that in both elderly males and females, moderate intensity exercise bouts lasting $<10 \mathrm{~min}$ three times a day for 24 weeks improved BP, $\mathrm{HR}$ and $\dot{V} \mathrm{O}_{2} \max$, and improvement was comparable to exercise bouts of $\geq 30 \mathrm{~min}$ as currently administered in 3-5 days a week. As adherence to exercise is a major factor in regular exercise, our findings could be of benefit to older men and women who face challenge of finding time for long continuous exercise sessions yet seek to improve their maximal oxygen uptake and cardiovascular parameters, improving their health.

\section{CONCLUSIONS}

For sedentary individuals aged $\geq 50$ years, moderate intensity exercise done in three intermittent daily sessions of $<10$ min each is as effective in the regulation of $\mathrm{BP}$ and improvement of maximal oxygen consumption as the currently recommended regime of 3-5 weekly exercise sessions of similar intensity lasting $\geq 30$ min each.

\section{Twitter Karani Magutah @karaniMaguth}

Contributors KM helped in designing protocol, data collection, analysis and writing of the manuscript. Both KT and NT helped with designing of protocol and writing the manuscript.

Funding This research was supported by the Consortium for Advanced Research Training in Africa (CARTA). CARTA is jointly led by the African Population and Health Research Center (APHRC) and the University of the Witwatersrand and funded by the Wellcome Trust (UK) (Grant No: 087547/Z/08/Z), the Department for International Development (DfID) under the Development Partnerships in Higher Education (DelPHE), the Carnegie Corporation of New York (Grant No: B 8606), the Ford Foundation (Grant No: 1100-0399), Google.Org (Grant No: 191994), Sida (Grant No: 54100029), MacArthur Foundation (Grant No: 10-95915-000-INP) and British Council.

\section{Competing interests None declared.}

Patient consent for publication Not required.

Ethics approval Study approval was granted by Moi Teaching and Referral Hospital/Moi University institutional research board.

Provenance and peer review Not commissioned; externally peer reviewed.

Data availability statement Data are available on reasonable request.

Open access This is an open access article distributed in accordance with the Creative Commons Attribution Non Commercial (CC BY-NC 4.0) license, which permits others to distribute, remix, adapt, build upon this work non-commercially, and license their derivative works on different terms, provided the original work is properly cited, appropriate credit is given, any changes made indicated, and the use is non-commercial. See: http://creativecommons.org/licenses/by-nc/4.0/.

\section{ORCID iD}

Karani Magutah http://orcid.org/0000-0003-3105-2981

\section{REFERENCES}

1 Whelton PK, Carey RM, Aronow WS, et al. 2017 ACC/AHA/AAPA ABC/ACPM/AGS/APhA/ASH/ASPC/NMA/PCNA guideline for the prevention, detection, evaluation, and management of high blood pressure in adults: Executive summary: a report of the American College of Cardiology/American heart association Task force on clinical practice guidelines. Hypertension 2018;71:1269-324.

2 Whelton PK, Carey RM, Aronow WS, et al. 2017 ACC/AHA/AAPA/ ABC/ACPM/AGS/APhA/ASH/ASPC/NMA/PCNA guideline for the prevention, detection, evaluation, and management of high blood pressure in adults: a report of the American College of Cardiology/ American heart association Task force on clinical practice guidelines. $J$ Am Coll

3 Nambaka JE, Kamau J, Amusa LO, et al. Factors influencing participation in physical exercise by the elderly in Eldoret West district, Kenya. African Journal for Physical, Health Education, Recreation and Dance 2011;17:462-72.

4 Sedentary Behaviour Research Network. Letter to the editor: standardized use of the terms "sedentary" and "sedentary behaviours". Appl Physiol Nutr Metab 2012;37:540-2. 
5 Kenya National Bureau of Statistics (KNBS), ICF Macro. Kenya Demoraphic and Health Survey 2008-09. Cavelton, Maryland: KNBS and ICF Micro, 2010.

6 Hendriks ME, Wit FWNM, Roos MTL, et al. Hypertension in subSaharan Africa: cross-sectional surveys in four rural and urban communities. PLoS One 2012;7:e32638.

7 BeLue R, Okoror TA, Iwelunmor J, et al. An overview of cardiovascular risk factor burden in sub-Saharan African countries: a socio-cultural perspective. Global Health 2009;5:10.

8 Ikem I, Sumpio BE. Cardiovascular disease: the new epidemic in sub-Saharan Africa. Vascular 2011;19:301-7.

9 World Health Organization. Global recommendations on physical activity for health 2010

10 Talbot LA, Morrell CH, Metter EJ, et al. Comparison of cardiorespiratory fitness versus leisure time physical activity as predictors of coronary events in men aged $<$ or $=65$ years and $>65$ years. Am J Cardiol 2002;89:1187-92.

11 DeBusk RF, Stenestrand U, Sheehan M, et al. Training effects of long versus short bouts of exercise in healthy subjects. Am J Cardiol 1990;65:1010-3.

12 Macfarlane DJ, Taylor LH, Cuddihy TF. Very short intermittent vs continuous bouts of activity in sedentary adults. Prev Med 2006;43:332-6.

13 Blair SN, Kohl HW, Gordon NF, et al. How much physical activity is good for health? Annu Rev Public Health 1992;13:99-126.

14 Pate RR, Pratt M, Blair SN, et al. Physical activity and public health. A recommendation from the centers for disease control and prevention and the American College of sports medicine. JAMA 1995;273:402-7.

15 LaFontaine T, Robbins L. Do multiple short bouts of exercise really produce the same benefits as single long bouts? Am J Cardiol 1991;67:325-6.

16 Murphy MH, Blair SN, Murtagh EM. Accumulated versus continuous exercise for health benefit: a review of empirical studies. Sports Med 2009;39:29-43.

17 Linke SE, Gallo LC, Norman GJ. Attrition and adherence rates of sustained vs. intermittent exercise interventions. Ann Behav Med 2011;42:197-209.

18 Magutah K, Meiring R, Patel NB, et al. Effect of short and long moderate-intensity exercises in modifying cardiometabolic markers in sedentary Kenyans aged 50 years and above. BMJ Open Sport Exerc Med 2018:4:e00316.

19 Magutah K, Patel NB, Thairu K. Effect of moderate-intensity exercise bouts lasting $<10$ minutes on body composition in sedentary Kenyan adults aged $\geq 50$ years. BMJ Open Sport Exerc Med 2018;4:e000403.

20 Fujimoto N, Hastings JL, Carrick-Ranson G, et al. Cardiovascular effects of 1 year of alagebrium and endurance exercise training in healthy older individuals. Circ Heart Fail 2013;6:1155-64.

21 Fujimoto N, Prasad A, Hastings JL, et al. Cardiovascular effects of 1 year of progressive and vigorous exercise training in previously sedentary individuals older than 65 years of age. Circulation 2010;122:1797-805.

22 Jackson AS, Sui X, Hébert JR, et al. Role of lifestyle and aging on the longitudinal change in cardiorespiratory fitness. Arch Intern Med 2009;169:1781-7.

23 Carson AP, Howard G, Burke GL, et al. Ethnic differences in hypertension incidence among middle-aged and older adults. Hypertension 2011;57:1101-7.

24 Vasan RS, Beiser A, Seshadri S, et al. Residual lifetime risk for developing hypertension in middle-aged women and men: the Framingham heart study. JAMA 2002;287:1003-10.

25 Global physical activity questionnaire (GPAQ) analysis guide. Available: http://www.who.int/chp/steps/resources/GPAQ_Analysis Guide.pdf [Accessed 01 May 2018].

26 Ramsbottom R, Brewer J, Williams C. A progressive shuttle run test to estimate maximal oxygen uptake. Br J Sports Med 1988;22:141-4.

27 CDC, 2015. Available: https://www.cdc.gov/physicalactivity/basics/ measuring/heartrate.htm [Accessed 14 Sep 2018].

28 Heyward HV. The Physical Fitness Specialist Certification Manual, The Cooper Institute for Aerobics Research, Dallas TX, revised 1997 printed in Advance Fitness Assessment \& Exercise Prescription. 3rd Ediition ed, 1998

29 Nonogi $\mathrm{H}$, Hess OM, Ritter M, et al. Diastolic properties of the normal left ventricle during supine exercise. Br Heart J 1988;60:30-8.

30 Alberti KGMM, Eckel RH, Grundy SM, et al. Harmonizing the metabolic syndrome: a joint interim statement of the International diabetes Federation Task force on epidemiology and prevention; National heart, lung, and blood Institute; American heart association; world heart Federation; international atherosclerosis Society; and international association for the study of obesity. Circulation 2009;120:1640-5.

31 WHO. Waist circumference and waist-hip ratio: report of a who expert consultation. Geneva, 2008: 8-11.

32 Lakka TA, Laaksonen DE, Lakka H-M, et al. Sedentary lifestyle, poor cardiorespiratory fitness, and the metabolic syndrome. Med Sci Sports Exerc 2003;35:1279-86.

33 Njelekela MA, Mpembeni R, Muhihi A, et al. Gender-Related differences in the prevalence of cardiovascular disease risk factors and their correlates in urban Tanzania. BMC Cardiovasc Disord 2009;9:30.

34 Tsimploulis A, Doumas M, Pittaras A, et al. YIA 01-06 cardiorespiratory fitness and mortality risk in hypertensive men $\geq 70$ years. J Hypertens 2016;34:e37-8.

35 Kokkinos P, Myers J, Kokkinos JP, et al. Exercise capacity and mortality in black and white men. Circulation 2008;117:614-22.

36 Kokkinos P, Puneet N, Apostolos T, et al. Os 04-09 cardiorespiratory fitness and risk for developing heart failure in hypertensives. $J$ Hypertens 2016;34:e57.

37 Juraschek SP, Blaha MJ, Whelton SP, et al. Physical fitness and hypertension in a population at risk for cardiovascular disease: the Henry Ford exercise testing (fit) project. J Am Heart Assoc 2014;3:e001268.

38 Al-Mallah MH, Juraschek SP, Whelton S, et al. Sex differences in cardiorespiratory fitness and all-cause mortality: the Henry Ford exercise testing (fit) project. Mayo Clin Proc 2016;91:755-62.

39 Landram MJ, Utter AC, Baldari C, et al. Differential effects of continuous versus discontinuous aerobic training on blood pressure and hemodynamics. J Strength Cond Res 2018;32:97-104.

40 Quinn TJ, Klooster JR, Kenefick RW. Two short, daily activity bouts vs. one long bout: are health and fitness improvements similar over twelve and twenty-four weeks? J Strength Cond Res 2006;20:130-5.

41 Peçanha T, Silva-Júnior ND, Forjaz CLdeM. Heart rate recovery: autonomic determinants, methods of assessment and association with mortality and cardiovascular diseases. Clin Physiol Funct Imaging 2014;34:327-39.

42 Dhoble A, Lahr BD, Allison TG, et al. Cardiopulmonary fitness and heart rate recovery as predictors of mortality in a referral population. J Am Heart Assoc 2014;3:e000559.

43 Benetos A, Safar M, Rudnichi A, et al. Pulse pressure: a predictor of long-term cardiovascular mortality in a French male population. Hypertension 1997;30:1410-5.

44 Gu Y-M, Aparicio LS, Liu Y-P, et al. Risk associated with pulse pressure on Out-of-Office blood pressure measurement. Pulse 2014;2:42-51.

45 Aparicio LS, Thijs L, Asayama K, et al. Reference frame for home pulse pressure based on cardiovascular risk in 6470 subjects from 5 populations. Hypertens Res 2014;37:672-8.

46 Sikiru L, Okoye GC. Effect of interval training programme on pulse pressure in the management of hypertension: a randomized controlled trial. Afr Health Sci 2013;13:571-8.

47 Dalleck LC, Borresen EC, Wallenta JT, et al. A moderate-intensity exercise program fulfilling the American College of sports medicine net energy expenditure recommendation improves health outcomes in premenopausal women. J Strength Cond Res 2008;22:256-62.

48 Gormley SE, Swain DP, High R, et al. Effect of intensity of aerobic training on VO2max. Med Sci Sports Exerc 2008;40:1336-43.

49 GladMohesh M, Sundaramurthy A. Effect of moderate exercise on VO2 max and blood pressure in individuals with different body mass index. J Clin Exp Res 2015;3:177-9.

50 Shephard RJ. Aging and Exercise. In: Fahey TD, ed. Encyclopedia of sports medicine and science. Internet Society for Sport Science, 1998. http://sportsci.org 